\title{
Synthesis of medium-chain glycerides using lipase from Candida rugose
}

\begin{abstract}
Enzymatic synthesis of medium-chain glycerides (MCG) from capric acid and glycerol was studied using lipase from Candida rugosa. The effects of various reaction parameters such as time, molar ratio of substrates ( $\mathrm{mmol}$ capric acid/mmol glycerol), amount of lipase, type of organic solvents, and initial water activity ( $\mathrm{a} \mathrm{w}$ ) were studied. The best conditions tested for MCG synthesis at $37^{\circ} \mathrm{C}$ were, respectively, time, $24 \mathrm{~h}$; molar ratio of substrates, 2.5; and amount of lipase, $100.0 \mathrm{mg}$. The use of organic solvents greatly influenced the activity of lipase in the synthesis of MCG. Generally, activity of lipase was high in nonpolar solvents with $\log \mathrm{P}$ values from 3.50 to 4.50 , where $\mathrm{P}$ is the partition coefficient between water and 1octanol. The enzymatic synthesis of MCG was preferably carried out at an initial a w of 0.328 , which resulted in maximal yield. Analysis of the products of reaction using gas chromatography showed that lipase from Candida rugosa seemed to produce more dicaprin and tricaprin than monocaprin.
\end{abstract}

Keyword: Esterification; Lipase; Medium-chain glycerides; Solvents; Water activity 